\title{
Stability of Superflow for Ultracold Fermions in Optical Lattices
}

\author{
A. A. Burkov \\ Department of Physics and Astronomy, University of Waterloo, Waterloo, Ontario, Canada N2L 3G1 \\ Arun Paramekanti \\ Department of Physics, University of Toronto, Toronto, Ontario, Canada M5S 1A7
}

(Dated: October 29, 2018)

\begin{abstract}
Motivated by recent observations of superfluidity of ultracold fermions in optical lattices, we investigate the stability of superfluid flow of paired fermions in the lowest band of a strong optical lattice. For fillings close to one fermion per site, we show that superflow breaks down via a dynamical instability leading to a transient density wave. At lower fillings, there is a distinct dynamical instability, where the superfluid stiffness becomes negative; this evolves, with increasing pairing interaction, from the fermion pair breaking instability to the well-known dynamical instability of lattice bosons. Our most interesting finding is the existence of a transition, over a range of fillings close to one fermion per site, from the fermion depairing instability to the density wave instability as the strength of the pairing interaction is increased.
\end{abstract}

One of the fundamental nonequilibrium properties of a superfluid is the critical velocity beyond which superflow breaks down. A closely related quantity is the critical flow momentum, $Q_{c}$, defined as the maximum sustainable phase gradient in the superfluid. This critical momentum conveys information about important length scales in the superfluid as is easily seen for dilute quantum gases. For dilute bosonic superfluids, the Landau criterion tells us that superfluidity breaks down when the flow velocity exceeds the velocity of the Bogoliubov 'phonons' of the superfluid. The critical flow momentum is then easily shown to be the inverse healing length of the superfluid. For weakly paired fermionic superfluids the superflow is limited by the small pairing gap. In this BCS regime, fermions depair at a critical flow momentum, which is the inverse Cooper pair size. As one tunes the interaction between fermions in a trapped Fermi gas through the BCS to BEC crossover, the critical momentum evolves from the depairing momentum of fermions to the inverse healing length of molecular bosons [1, 2].

A different route to the breakdown of superfluidity is through a dynamical instability as has been experimentally observed for bosonic atoms in optical lattices [3]. For weakly correlated bosons in a lattice, there is a critical momentum, $Q_{c} \sim \hbar(\pi / 2 a)$, where $a$ is the optical lattice constant, at which superflow breaks down as the effective superfluid density becomes negative [4]. (Henceforth we will set $\hbar=1, a=1$.) For bosons at commensurate filling this dynamical instability has been shown $[\underline{5}, \underline{6}$ ] to be smoothly connected to the equilibrium superfluid to Mott insulator transition of bosons [7], in the sense that the critical momentum for the onset of this dynamical instability tends to zero as the system is tuned towards the Mott transition. Close to the Mott transition, $Q_{c} \sim 1 / \xi$, where $\xi$ is the diverging correlation length for fluctuations near the superfluid to Mott insulator transition. Thus,

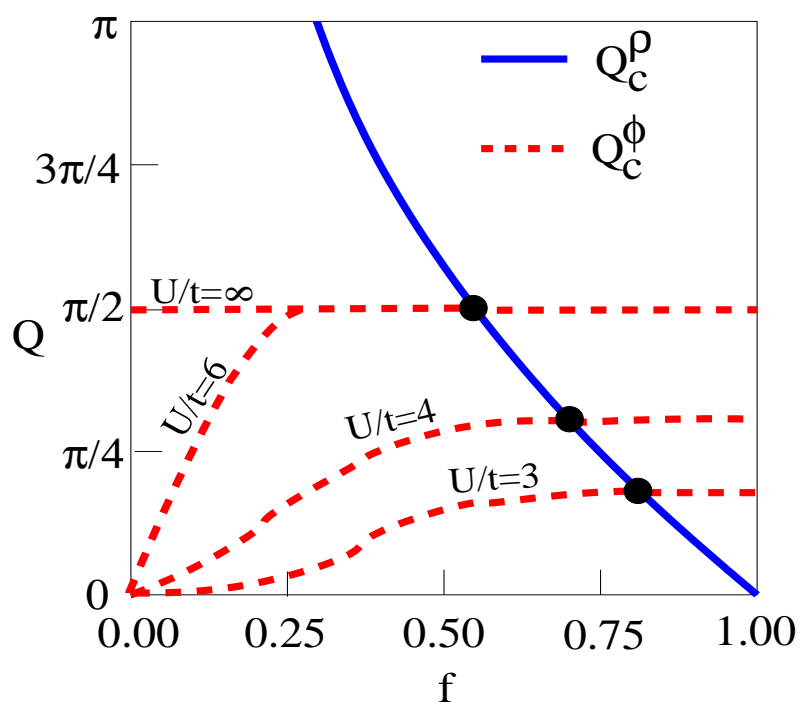

FIG. 1: (Color online) Critical flow momenta for phase stiffness dynamical instability, $Q_{c}^{\phi}$, and density wave dynamical instability, $Q_{c}^{\rho}$, versus fermion filling $f$ at various values of $\mathrm{U} / \mathrm{t}$ in three dimensions [8]. For $Q_{c}^{\phi} \lesssim \pi / 2$, the fermions depair at $Q \approx Q_{c}^{\phi}$, but they depair at larger values of $Q$ once $Q_{c}^{\phi}=\pi / 2$. Black dots indicate points where a sharp transition from the phase stiffness to the density wave instability occurs.

the critical flow momentum in this case conveys nontrivial information about an equilibrium quantum phase transition.

In this Letter, we focus on the remarkable and rich physics associated with the breakdown of superflow of paired fermions in an optical lattice. In the strong lattice potential limit, the ultracold fermion system can be described by a single band negative- $U$ Hubbard model 9], which we define on a $d$-dimensional hypercubic lat- 
tice:

$$
H_{\mathrm{HUB}}=-\sum_{i, j} t_{i j} c_{i \sigma}^{\dagger} c_{j \sigma}-\mu \sum_{i} n_{i}-U \sum_{i} n_{i \uparrow} n_{i \downarrow},
$$

where $c_{i \sigma}^{\dagger}$ creates a fermionic atom in the hyperfine spin state $\sigma$ on a lattice site $i$. This model is well known to have a superfluid ground state. The phase diagram in Fig. 1 summarizes the two main new results of our work. (i) For fillings far from one fermion per site $(f=1)$, we show that there is a dynamical instability at a critical momentum $Q_{c}^{\phi}$ where the effective superfluid density becomes negative leading to the breakdown of superflow. For large $U / t$, this is related to the dynamical instability of lattice bosons, discussed earlier, while for small $U / t$ it is closely related to depairing of fermions. (ii) We show that for $f=1$, there is an insulating crystal state which is degenerate (or nearly degenerate) with the uniform superfluid. For a range of fillings around $f=1$, we show that this competing crystal state leads to the breakdown of superflow via a distinct dynamical instability at $Q_{c}^{\rho}$, which leads to a transient density wave as shown in Fig. 2. The critical momentum for this instability is the inverse density correlation length (at the relevant wavevector) in the uniform superfluid.

To observe this dynamical instability in cold atom experiments, two ingredients are necessary. First, one needs counterpropagating laser beams, which are frequencydetuned from each other to generate a moving optical lattice. Indeed, a recent experiment using such moving lattices has successfully measured the critical current of paired fermions [1] in a weak optical lattice. Second, one needs to be able to detect the density wave order, induced by the dynamical instability. This could be done using light scattering or noise [10] measurements. Light scattering at intermediate timescales should be able to detect broadened Bragg spots associated with the emergent crystalline order.

While our work is in part inspired by recent experiments demonstrating fermionic superfluidity in an optical lattice [11], a broader motivation is to explore how competing broken symmetry states, such as charge density waves, lead to a breakdown of superflow in uniform strongly correlated superfluids - this issue is of great importance for systems ranging from high temperature superconductors [12] to excitonic superfluids in quantum Hall bilayers [13].

We begin with the observation [14] that if the fermions only hop between nearest neighbor sites and if the density is fixed to be one fermion per site $(f=1)$, the Hubbard model Eq.(11) possesses an extra pseudospin $\mathrm{SU}(2)$ symmetry in addition to the usual $\mathrm{SU}(2)$ spin rotation symmetry. Let us define the local version of Anderson's pseudospin operators [15] as $T_{i}^{+}=c_{i \uparrow}^{\dagger} c_{i \downarrow}^{\dagger}, T_{i}^{-}=c_{i \downarrow} c_{i \uparrow}, T_{i}^{z}=$ $\frac{1}{2}\left(\sum_{\sigma} c_{i \sigma}^{\dagger} c_{i \sigma}-1\right)$. One can show that the total pseudospin operators $\sum_{i} s_{i} T_{i}^{ \pm}, \sum_{i} T_{i}^{z}$ commute with the negative- $U$
Hubbard Hamiltonian, where $s_{i}=+1,-1$ on the two sublattices of the hypercubic lattice. This leads to an exact degeneracy between the superfluid and the "checkerboard" $(\pi, \ldots, \pi)$ charge density wave (CDW) states at $f=1$. In pseudospin language, the superfluid corresponds to ferromagnetic ordering of $T^{ \pm}$while the CDW corresponds to antiferromagnetic ordering of $T^{z}$.

This degeneracy, and what lifts it, is most clearly revealed in the large $U / t$ limit of Eq.(11), which can be realized in cold atom experiments by going to a stronger optical lattice potential. In this limit, we can view the presence (absence) of a fermion pair at a site as a pseudospin up (down) configuration, and the Hamiltonian in Eq.(11) maps onto a spin-a-half pseudospin model:

$$
H_{\mathrm{xxz}}=\sum_{i, j} \frac{J_{i j}}{2}\left[T_{i}^{z} T_{j}^{z}-\frac{1}{2}\left(T_{i}^{+} T_{j}^{-}+\text {h.c. }\right)\right]-2 \mu \sum_{i} T_{i}^{z},
$$

where $J_{i j}=4 t_{i j}^{2} / U$. At $\mu=0$, which corresponds to $f=2\left\langle T_{i}^{z}\right\rangle+1=1$, and assuming $J_{i j}$ connects only neighboring sites with a strength $J \equiv 4 t^{2} / U$, Eq.(2) has $\mathrm{SU}(2)$ pseudospin symmetry.

This pseudospin $\mathrm{SU}(2)$ symmetry is broken in favor of the superfluid for nonzero $\mu$, which dopes the system away from $f=1$. It is lifted even when $\mu=0$ if the pseudospin exchange $J_{i j}$ connects next neighbor sites, with a magnitude $J^{\prime} \equiv 4 t^{\prime 2} / U \ll J$, in which case the 'classical' ferromagnetic state of $T^{ \pm}$has a lower energy density, compared to the antiferromagnetic state of $T^{z}$, by an amount proportional to $J^{\prime}$. The question we would like to address here is how the presence of this broken symmetry crystal as a competing ordered state close by in energy leads to an instability of the uniform flowing superfluid. We begin with a large $U / t$ analysis, where the physics is fully described by our pseudospin model Eq.(2); we then generalize our results to all values of $U / t$. For simplicity we set $t^{\prime}=0$, the $t^{\prime} \neq 0$ case is not substantially different.

The evolution of the metastable current-carrying state of (3) as a function of the filling $f$ and the flow momentum $Q$ can be conveniently analyzed in the semiclassical limit of large pseudospin. Let $H_{c \ell}$ denote the classical Hamiltonian, obtained by replacing the pseudospin operators in $H_{\mathrm{xxz}}$ by angular momentum vectors $\vec{T}_{i} \equiv T \vec{\Omega}_{i}$, where $\vec{\Omega}_{i}=\left(\cos \theta_{i}, \sin \theta_{i} \cos \phi_{i}, \sin \theta_{i} \sin \phi_{i}\right)$ are unit vectors and $T$ denotes the magnitude of the angular momentum $(T=1 / 2$ in our case). With this parametrization,

$$
\begin{aligned}
H_{c \ell}[\Omega] & =\frac{T^{2}}{2} \sum_{i j} J_{i j}\left[\cos \theta_{i} \cos \theta_{j}-\sin \theta_{i} \sin \theta_{j} \cos \left(\phi_{i}-\phi_{j}\right)\right] \\
& -2 \mu T \sum_{i} \cos \left(\theta_{i}\right) .
\end{aligned}
$$

For brevity, we present analytical results for $f \leq 1$; the physics is identical for $f>1$ when $J^{\prime}=0$.

Assuming the current flows in the $x$-direction, we begin by extremizing the classical energy to find $\theta_{i}=\theta=$ 
$\arctan [\sqrt{f(2-f)} /(f-1)], \phi_{i}=Q x_{i}, \mu=d J T(f-$ $1)\left(1+\gamma_{\mathbf{Q}}\right)$, where $\gamma_{\mathbf{k}}=\frac{1}{d} \sum_{i=1, \ldots, d} \cos \left(k_{i}\right)$ and we have assumed that the filling $f$, rather than the chemical potential, is fixed. Stability of the superflow depends on whether this state is a local minimum of the energy functional or just a saddle point. To answer this question we expand $H_{c \ell}$ to second order in fluctuations about the above solution and Fourier transform it to obtain:

$$
\begin{aligned}
H_{c \ell}[\Omega] & =\sum_{\mathbf{k}}\left[\rho_{\theta \theta}(\mathbf{k}) \delta \theta_{\mathbf{k}}^{*} \delta \theta_{\mathbf{k}}+\rho_{\phi \phi}(\mathbf{k}) \delta \phi_{\mathbf{k}}^{*} \delta \phi_{\mathbf{k}}\right. \\
& \left.+\rho_{\theta \phi}(\mathbf{k}) \delta \theta_{\mathbf{k}}^{*} \delta \phi_{\mathbf{k}}+\rho_{\theta \phi}^{*}(\mathbf{k}) \delta \phi_{\mathbf{k}}^{*} \delta \theta_{\mathbf{k}}\right],
\end{aligned}
$$

where we have subtracted the energy of the uniform flowing state. Defining $\eta_{\mathbf{Q}}(\mathbf{k}) \equiv\left(\gamma_{\mathbf{k}+\mathbf{Q}}+\gamma_{\mathbf{k}-\mathbf{Q}}\right) / 2$, the three different stiffness coefficients in (4) are given by:

$$
\begin{aligned}
\rho_{\theta \theta}(\mathbf{k}) & =\frac{\partial^{2} H_{c \ell}}{\partial \theta_{\mathbf{k}}^{*} \partial \theta_{\mathbf{k}}}=d J T^{2}\left[\gamma_{\mathbf{Q}}-\eta_{\mathbf{Q}}(\mathbf{k})\right. \\
& \left.+\left(\gamma_{\mathbf{k}}+\eta_{\mathbf{Q}}(\mathbf{k})\right) \sin ^{2}(\theta)\right] \\
\rho_{\phi \phi}(\mathbf{k}) & =\frac{\partial^{2} H_{c \ell}}{\partial \phi_{\mathbf{k}}^{*} \partial \phi_{\mathbf{k}}}=d J T^{2} \sin ^{2}(\theta)\left(\gamma_{\mathbf{Q}}-\eta_{\mathbf{Q}}(\mathbf{k})\right) \\
\rho_{\theta \phi}(\mathbf{k}) & =\frac{\partial^{2} H_{c \ell}}{\partial \theta_{\mathbf{k}}^{*} \partial \phi_{\mathbf{k}}}=-i \frac{d J T^{2} \sin (2 \theta)}{4}\left(\gamma_{\mathbf{k}+\mathbf{Q}}-\gamma_{\mathbf{k}-\mathbf{Q}}\right) .
\end{aligned}
$$

Here $\rho_{\theta \theta}(\mathbf{k})$ is the inverse density susceptibility at wavevector $\mathbf{k}, \rho_{\phi \phi}(\mathbf{k})$ is the phase stiffness and $\rho_{\theta \phi}(\mathbf{k})$ is related to the current, transported by the mode $\mathbf{k}$.

The flowing state is a stable local minimum provided the following conditions are satisfied:

$$
\rho_{\theta \theta}(\mathbf{k}), \rho_{\phi \phi}(\mathbf{k})>0, \rho_{\theta \theta}(\mathbf{k}) \rho_{\phi \phi}(\mathbf{k})>\left|\rho_{\theta \phi}(\mathbf{k})\right|^{2}, \forall \mathbf{k} .
$$

There are thus three distinct instabilities, which can lead to the decay of superflow. For reasons that will become clear below, the instabilities corresponding to vanishing $\rho_{\theta \theta}$ or $\rho_{\phi \phi}$ are called dynamical, and we will refer to the critical flow momenta, where these occur, as $Q_{c}^{\rho}$ and $Q_{c}^{\phi}$ respectively, while instability corresponding to the violation of the last condition in Eq.(6) is called Landau or energetic instability [4] and we will refer to the critical flow momentum for this instability as $Q_{c}^{L a n}$.

Both dynamical and Landau instabilities have the same physical origin, namely the superflow-carrying state becoming a saddle-point of the energy functional instead of a local minimum. In order to understand the difference between these instabilities, we turn to an analysis of the dynamics. Defining new variables $p_{\mathbf{k}}=T \sin (\theta) \delta \theta_{\mathbf{k}}=$ $p_{1 \mathbf{k}}+i p_{2 \mathbf{k}}$ and $q_{\mathbf{k}}=\delta \phi_{\mathbf{k}}=q_{1 \mathbf{k}}+i q_{2 \mathbf{k}}$, it is easy to show that $p_{\nu \mathbf{k}}$ and $q_{\nu \mathbf{k}}$ are canonically conjugate variables which thus obey Hamiltonian quasiclassical equations of motion. Rewritten in terms of $p_{\nu}$ and $q_{\nu}$, the fluctuation Hamiltonian Eq.(44) has the form of a Hamiltonian of a set of decoupled harmonic oscillators in two dimensions, labelled by $\mathbf{k}$, with characteristic frequency $\omega_{0 \mathbf{k}}=2 \sqrt{\rho_{\theta \theta}(\mathbf{k}) \rho_{\phi \phi}(\mathbf{k})} / T \sin (\theta)$, rotating with frequency
$\Omega_{\mathbf{k}}=2\left|\rho_{\theta \phi}(\mathbf{k})\right| / T \sin (\theta)$. In this language the two dynamical instabilities correspond to the point where the frequency of one or more of the oscillators becomes imaginary, while Landau instability corresponds to the rotation frequency exceeding the oscillator frequency. Diagonalizing the Hamiltonian by a canonical transformation we obtain the eigenfrequencies $\omega_{\mathbf{k}}=\omega_{0 \mathbf{k}} \pm \Omega_{\mathbf{k}}$. Spectral stability requires $\rho_{\theta \theta}(\mathbf{k})>0, \rho_{\phi \phi}(\mathbf{k})>0$. When either one of these become negative, the eigenmode frequencies acquire imaginary parts, which means that isoenergetic orbits in the phase space become open, i.e. the motion in the neighborhood of the $p_{\mathbf{k}}=0, q_{\mathbf{k}}=0$ saddle point becomes dynamically unstable. The motion in the Landauunstable regime is, however, dynamically stable in this linearized problem, since isoenergetic orbits in this case are closed. Once mode-coupling, due to nonlinearities or external potentials, is included the motion will most likely immediately become unstable. We however expect that at low temperatures and in two and three dimensions, where quantum corrections to the quasiclassical dynamics are weak [16], the Landau instability in our system system will develop on much longer time scales compared to dynamical instabilities.

After this preliminary discussion of the instabilities, we now calculate the dynamical phase diagram of our system as a function of the flow momentum $Q$ and the filling $f$. Straightforward analysis of Eq.(15) gives the following results at $T=1 / 2$ :

$$
\begin{aligned}
\cos Q_{c}^{\phi}(f) & =0 \\
\cos Q_{c}^{\rho}(f) & =\frac{1-(2 d-1)(1-f)^{2}}{1+(1-f)^{2}}, \\
\cos Q_{c}^{\text {Lan }}(f) & =\frac{A(f, d)-(2 d-1) f(2-f)}{2\left[1+(1-f)^{2}\right]},
\end{aligned}
$$

where

$$
A(f, d) \equiv \sqrt{(2 d-1)^{2} f^{2}(2-f)^{2}+8(1-f)^{2}\left[1+(1-f)^{2}\right]} .
$$

Focussing on the dynamical instabilities, the most interesting feature of Eq.(7) is singular behavior of the critical momenta as functions of the fermion density. Defining $f^{*} \equiv 1-1 / \sqrt{2 d-1}$, we find that for $f<f^{*}, Q_{c}^{\phi}<Q_{c}^{\rho}$, while for $f>f^{*}, Q_{c}^{\rho}<Q_{c}^{\phi}$. There is thus a sharp transition, as a function of filling at $f^{*}$, from an instability due to a vanishing phase stiffness $\rho_{\phi \phi}(\mathbf{k})$ for $0<f<f^{*}$ to an instability due to a vanishing inverse susceptibility $\rho_{\theta \theta}(\mathbf{k})$ for $f^{*}<f<1$. In $d=1$, we find $f^{*}=0$. The phase-stiffness-related instability involves all wavevectors of the form $\mathbf{k}=\left(k_{x}, 0, \ldots, 0\right)$ (for flow in the $x$-direction). The density modulation dynamical instability, by contrast, occurs at a single wavevector $(\pi, \ldots, \pi)$, since this instability is directly related to the checkerboard CDW state. For $d=3$, this CDW related dynamical instability only exists for $1-1 / \sqrt{2}<f<1$.

An interesting question is what is the state, towards which the uniform flowing superfluid becomes unsta- 
ble, when $Q_{c}^{\rho}(f)<Q<Q_{c}^{\phi}(f)$. Since the instability is characterized by a divergent density susceptibility at $(\pi, \ldots, \pi)$, one might expect the resulting state to be a "flowing supersolid" [17, in which the CDW coexists with superfluid flow. Indeed, one finds that such a state extremizes the classical energy and has lower energy than the uniform state for $Q>Q_{c}^{\rho}$. Analyzing the stability of such a state, however, we find that it is in turn dynamically unstable due to a negative superfluid stiffness.

Since there does not appear to be a simple stable equilibrium state beyond the $(\pi, \ldots, \pi)$ dynamical instability, we numerically integrate the semiclassical LandauLifshitz equations, $\frac{d \vec{T}_{i}}{d t}=\left\{H_{c \ell}, \vec{T}_{i}\right\}+\eta \vec{T}_{i} \times\left\{H_{c \ell}, \vec{T}_{i}\right\}$, where \{\} denotes the Poisson bracket, in order to understand the fate of the system for $Q>Q_{c}^{\rho}$. We assume a phenomenological damping term, $\eta \ll 1$, to mimic weak dissipation arising from thermal excitations, which are relevant for experiment but not taken into account in our approach. The density order, expected to emerge beyond $Q_{c}^{\rho}$, can be characterized by the density structure factor $S(G, t)=(4 / L) \sum_{i, m} T_{m}^{z}(t) T_{m+i}^{z}(t) \exp \left(-i G x_{i}\right)$. To illustrate the nature of the collective many body dynamics associated with the density modulation instability, we focus on two physical observables: the density order parameter, $\rho_{G}(t) \equiv \sqrt{S(G, t) / L}$, at the wavevector $G$ where $S(G, t)$ is the largest at that time, and the fermion pair current $I_{s}(t)=(4 / L) \sum_{i}\left[T_{i}^{x}(t) T_{i+1}^{y}(t)-T_{i}^{y}(t) T_{i+1}^{x}(t)\right]$. We observe (see Fig. 2) that starting from the uniform superfluid, there is an initial growth of a density modulation order parameter, with a growth rate proportional to $Q-Q_{c}^{\rho}$ and independent of the weak dissipation. The density modulation wavevector $G$ fluctuates around $\pi$, which is the ordering wavevector of the CDW crystal. The growth of this CDW order leads to a drop in the flow momentum as the current decays via phase slips in regions where the local density reaches $f=1$, giving rise to a transient insulating CDW patch. At these intermediate timescales, the CDW order is unstable as discussed and exhibits fluctuations over time scales $1 / J$ (arising from excited collective modes) as seen in Fig. 1. In the presence of dissipation, the system eventually settles down into a new (lower energy) steady state which, typically, is a uniformly flowing state, where the CDW order has decayed away and the final flow momentum is $Q<Q_{c}^{\rho}$. The main message from our numerics is that even when the "flowing supersolid" state is dynamically unstable, a transient density wave nevertheless emerges when $Q>Q_{c}^{\rho}$. For modest dissipation, its fluctuations persist over a time scale set by the inverse dissipation rate as seen from the Fig. 2 .

We now generalize the large $U / t$ results, discussed so far, to all values of $U / t$. For small fillings $f<f^{*}$ and large $U / t$ we see that the first dynamical instability one encounters is at $Q_{c}^{\phi}=\pi / 2$. However, for weaker couplings, one can view the Anderson pseudospin model as being applicable at length scales larger than the BCS co-

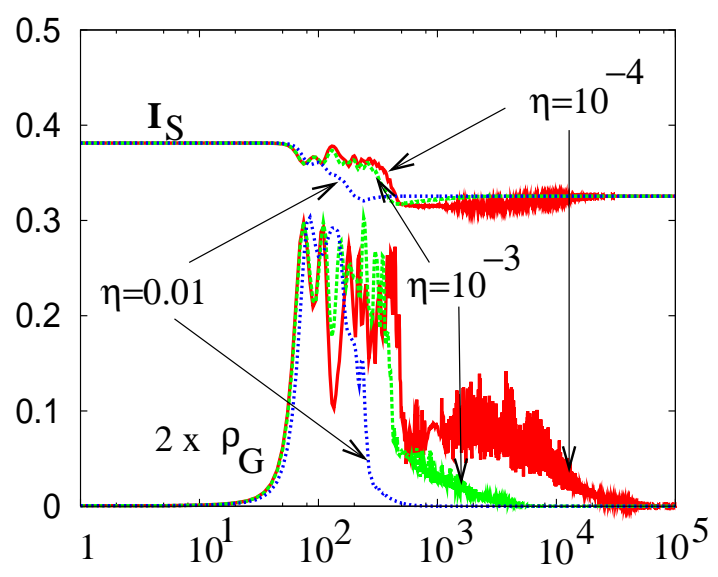

Time (units of $1 / \mathrm{J}$ )

FIG. 2: (Color online) Evolution of the current, $I_{s}$, and the scaled CDW order parameter, $2 \times \rho_{G}$, for a one dimensional lattice of 200 sites with $f=0.8$ (corresponding to $\left.Q_{c}^{\rho} \approx 0.125 \pi\right), Q=0.13 \pi$ and indicated values of dissipation.

herence length, which is proportional to the fermion pair size $\xi^{\text {pair }}$. Thus, this phase stiffness instability will occur at $Q_{c}^{\phi} \sim \pi / 2 \xi^{\text {pair }}$ instead of $\pi / 2$, where $\xi^{\text {pair }} \gg 1$ at weak coupling. From this result it is clear that, at weak coupling, this dynamical instability is simply related to the fermion depairing instability - once the flow momentum exceeds the inverse fermion pair size, the pairs will break up. Thus, when $f<f^{*}$, there is a long wavelength dynamical instability associated with vanishing $\rho_{\phi \phi}$, which smoothly evolves from the vicinity of the pair breaking instability $Q_{c}^{\phi} \sim 1 / \xi^{\text {pair }} \ll 1$ when $U / t \ll 1$, to $Q_{c}^{\phi}=\pi / 2$ when $U / t \gg 1$. At fermion densities $f>f^{*}$ the situation is completely different. In this case, for small $U / t$, there is still the above dynamical instability, which will occur close to the depairing instability. However, at larger $U / t$, this instability is preempted by the distinct density modulation dynamical instability at $Q_{c}^{\rho}$, which is set by the inverse correlation length of the incipient CDW order in the uniform superfluid. The transition between the depairing and the density wave instabilities occurs when $Q_{c}^{\rho} \xi^{\text {pair }} \sim 1$, i.e. when the fermion pair size becomes smaller than the correlation length of the CDW order. This correlation length diverges at $f=1$. Since these two dynamical instabilities involve completely different wavevectors and different susceptibilities, they can not be smoothly connected; thus for fillings $f^{*}<f<1$, there will be a sharp change in the character of the dynamical instability with increasing $U / t$. This sharp change should be contrasted with a smooth evolution of the ground state properties with increasing $U / t$ (BCS-BEC crossover). Our result implies that while the evolution of the ground state is smooth, the evolution of the excitation spectrum in the presence of competing ordered states nearby in energy is not smooth, which is revealed 
in the way the superfluid flow breaks down once the critical phase gradient is exceeded. We have confirmed the above heuristic arguments by generalizing the BCS mean field theory to include flow and density wave order parameter. The results of our calculations are summarized in Fig. 11 details will be presented elsewhere [18].

We thank E. Altman, E. Demler, A. Griffin, S. Morris, P. Nikolic, T. Sheppard and J. Thywissen for discussions. AAB was supported by a startup grant from the University of Waterloo. AP acknowledges support from NSERC and the Alfred P. Sloan foundation.

[1] D. E. Miller et al., Phys. Rev. Lett. 99, 070402 (2007).

[2] R. Combescot el al., Phys. Rev. A 74, 042717 (2006); R. Sensarma et al., Phys. Rev. Lett. 96, 090403 (2006); R.B. Diener et al., arXiv:0709.2653 (unpublished).

[3] L. Fallani et al., Phys. Rev. Lett. 93, 140406 (2004).

[4] B. Wu and Q. Niu, Phys. Rev. A 64, 061603(R) (2001).

[5] E. Altman et al., Phys. Rev. Lett. 95, 020402 (2005).

[6] J. Mun et al., Phys. Rev. Lett. 99, 150604 (2007).
[7] M. P. A. Fisher et al., Phys. Rev. B 40, 546 (1989); M. Greiner et al., Nature (London) 415, 39 (2002).

[8] The $Q_{c}^{\rho}$ in Fig. 1 has not been shown as a function of $U / t$ since the strong coupling limit gives a reasonable approximation for this instability point for all $U / t \gtrsim 3$. Details will be given in [18].

[9] R. Micnas et al., Rev. Mod. Phys. 62, 113 (1990); A. Koetsier et al., Phys. Rev. A 74, 033621 (2006); C.-C. Chien et al., ibid. 77, 011601(R) (2008).

[10] E. Altman et al., Phys. Rev. A 70, 013603 (2004); S. Foelling et al., Nature 434, 481 (2005).

[11] J.K. Chin et al., Nature (London) 443, 961 (2006).

[12] J.M. Tranquada et al., Nature (London) 429, 534 (2004).

[13] M. Abolfath et al., Phys. Rev. B 68, 155318 (2003).

[14] C.N. Yang and S.C. Zhang, Mod. Phys. Lett. B 4, 759 (1990).

[15] P.W. Anderson, Phys. Rev. 112, 1900 (1958).

[16] Here we disregard the possible broadening of instabilities in $d=1,2$ by quantum tunneling out of the metastable flowing state [5]. These issues will be addressed in [18].

[17] E. Zhao and A. Paramekanti, Phys. Rev. Lett. 96, 105303 (2006).

[18] G. Ramachandran et al., in preparation. 